\title{
Statistics of General Order Selection in Correlated Nakagami Fading Channels
}

\author{
Maged Elkashlan, Tamer Khattab, Cyril Leung, and Robert Schober
}

\begin{abstract}
In this letter, the cumulative distribution function (and hence outage probability) of the $r$-th order signal-to-noise ratio from a set of $n$ correlated Nakagami fading branches is studied. Numerical results are presented to illustrate the effect of fading correlation and the fading severity parameter. The accuracy of a simple exchangeable approximation is also examined.
\end{abstract}

Index Terms-Correlated fading, diversity, Nakagami fading, order statistics.

\section{INTRODUCTION}

$\mathbf{I}$ $\mathrm{N}$ the design of wireless communication systems, much research effort has been devoted to the study of diversity schemes for mitigating the effects of channel fading and improving the received signal-to-noise ratio (SNR). The theory of order statistics underpins the performance analysis of many diversity reception techniques. Selection combining (SC) is a simple diversity scheme in which the signal from the branch with the highest SNR is processed. In generalized selection combining (GSC), the received signals on the $L$ branches with highest SNR values are combined [1], [2]. Most studies of diversity systems assume that the fading processes on the different branches are uncorrelated. The performance analysis of systems involving selection combining and correlated fading has been considered in [2]-[6] and the citations therein.

In signal detection and estimation problems, the cumulative distribution function (cdf) of the $r$-th order statistic is often required. Applications in which this cdf is useful include: (1) the evaluation of the performance loss when the receiver makes an error in selecting the largest SNR branch, (2) the calculation of outage capacity and of the average symbol error rates for various modulation schemes, and (3) the performance analysis of order selection diversity, in which the $r$-th order branch is selected for transmission.

Expressions for the cdf of the $r$-th order statistic, $r=$ $1,2, \ldots, n$, for correlated Nakagami fading branches have not appeared in the literature except for $r=n$ (i.e. the largest order statistic). In this letter, we apply a general result from the theory of order statistics [7] for evaluating the cdf of the $r$-th order random variable (rv) out of a set of $n$ arbitrarily correlated rv's to the case of correlated Nakagami- $m$ fading. Specifically, numerical results are obtained which illustrate the

Paper approved by M. Chiani, the Editor for Wireless Communication of the IEEE Communications Society. Manuscript received December 23, 2005; revised October 20, 2006. This paper was presented in part at the IEEE Pacific Rim Conference on Communications, Computers and Signal Processing, Victoria, BC, Canada, Aug. 2005.

The authors are with the Department of Electrical and Computer Engineering, The University of British Columbia, Vancouver, BC, Canada V6T 1Z4 (e-mail: \{magede, tkhattab, cleung, rschober\}@ece.ubc.ca)

Digital Object Identifier 10.1109/TCOMM.2008.050646. effects of the fading parameter $m$ and the branch correlation matrix. For a large number of branches, the numerical procedure can be quite computationally intensive. Thus, the accuracy of using a simplifying exchangeable ${ }^{1}$ approximation is also investigated.

\section{RELEVANT CDF's}

Let $G_{1}, G_{2}, \cdots, G_{n}$ be $n$ arbitrarily correlated rv's which represent the branch gains in a diversity communication system. The corresponding branch SNR's are denoted by $\Gamma_{1}, \Gamma_{2}, \cdots, \Gamma_{n}$, with

$$
\Gamma_{i} \triangleq\left[G_{i}\right]^{2} E / N_{0}, i=1,2, \ldots, n
$$

where $E$ is the transmitted bit energy and $N_{0}$ is the one-sided noise power spectral density (PSD).

If the rv's $G_{1}, G_{2}, \cdots, G_{n}$ are arranged in increasing order of their magnitudes and written as

$$
G_{1: n} \leq G_{2: n} \leq \cdots \leq G_{n: n}
$$

we refer to $G_{r: n}$, as the $r$-th order statistic.

The joint cdf of $\left(\Gamma_{1}, \cdots, \Gamma_{n}\right)$ can be expressed as [8, p. 140]

$$
\begin{aligned}
F_{\Gamma_{1}, \cdots, \Gamma_{n}}\left(\gamma_{1}, \cdots, \gamma_{n}\right) & =\frac{1}{(2 \pi)^{n}} \int_{-\infty}^{\infty} \cdots \int_{-\infty}^{\infty} \phi\left(t_{1}, \cdots, t_{n}\right) \\
& \times \prod_{k=1}^{n}\left(\frac{1-e^{-j t_{k} \gamma_{k}}}{j t_{k}}\right) d t_{1} \cdots d t_{n},(3)
\end{aligned}
$$

where $\phi\left(t_{1}, \cdots, t_{n}\right)$ is the joint characteristic function (CF) of $\left(\Gamma_{1}, \cdots, \Gamma_{n}\right)$. Hence, the cdf of the highest order statistic $\Gamma_{n: n} \triangleq \max \left\{\Gamma_{1}, \cdots, \Gamma_{n}\right\}$ can be written as shown in (4).

From [7, p. 99], the cdf of the $r$-th order statistic can be obtained as shown in (5). In (5), the inner sum is over all possible ways of selecting $j$ out of $n$ rv's and the superscript notation in $F_{\Gamma_{j: j}}^{\left(i_{1}, \cdots, i_{j}\right)}$ indicates that only $\Gamma_{i_{1}}, \cdots, \Gamma_{i_{j}}$ are included in the selection. It is assumed that the inner sum is equal to $F_{\Gamma_{n: n}}(\gamma)$ for $j=n$. The term $F_{\Gamma_{j: j}}^{\left(i_{1}, \cdots, i_{j}\right)}(\gamma)$ is obtained using (4).

When $n$ is large, the evaluation of (5) may be timeconsuming. However, if the $\Gamma_{i}$ 's are exchangeable, (5) can be easily evaluated as it reduces to

$$
F_{\Gamma_{r: n}}(\gamma)=\sum_{j=r}^{n}(-1)^{j-r}\left(\begin{array}{l}
j-1 \\
r-1
\end{array}\right)\left(\begin{array}{l}
n \\
j
\end{array}\right) F_{\Gamma_{j: j}}(\gamma)
$$

\footnotetext{
${ }^{1}$ A set of random variables $A_{1}, \cdots, A_{n}$ are said to be exchangeable if the joint distribution of $A_{\pi_{1}}, \cdots, A_{\pi_{n}}$ does not depend on the permutation $\pi$.
} 


$$
\begin{aligned}
F_{\Gamma_{n: n}}(\gamma) & =\operatorname{Pr}\left\{\text { all } \Gamma_{i} \leq \gamma\right\} \\
& =\frac{1}{(2 \pi)^{n}} \int_{-\infty}^{\infty} \cdots \int_{-\infty}^{\infty} \phi\left(t_{1}, \cdots, t_{n}\right) \prod_{k=1}^{n}\left(\frac{1-e^{-j t_{k} \gamma}}{j t_{k}}\right) d t_{1} \cdots d t_{n} \\
F_{\Gamma_{r: n}}(\gamma) & =\sum_{j=r}^{n}\left[(-1)^{j-r}\left(\begin{array}{l}
j-1 \\
r-1
\end{array}\right) \sum_{1 \leq i_{1}<\cdots<i_{j} \leq n} F_{\Gamma_{j: j}}^{\left(i_{1}, \cdots, i_{j}\right)}(\gamma)\right]
\end{aligned}
$$

If $G_{1}, G_{2}, \cdots, G_{n}$ are independent, but not necessarily identically distributed rv's, (4) simplifies to

$$
\begin{aligned}
F_{\Gamma_{n: n}}(\gamma) & =\operatorname{Pr}\left\{\Gamma_{1}, \cdots, \Gamma_{n} \leq \gamma\right\} \\
& =\operatorname{Pr}\left\{\Gamma_{1} \leq \gamma\right\} \cdots \operatorname{Pr}\left\{\Gamma_{n} \leq \gamma\right\} \\
& =\prod_{k=1}^{n} F_{\Gamma_{k}}(\gamma) .
\end{aligned}
$$

Substituting (7) into (5) we obtain (8).

In the special case when $G_{1}, G_{2}, \cdots, G_{n}$ are independent, identically distributed (i.i.d.) rv's, (8) reduces to

$$
F_{\Gamma_{r: n}}(\gamma)=\sum_{j=r}^{n}(-1)^{j-r}\left(\begin{array}{l}
j-1 \\
r-1
\end{array}\right)\left(\begin{array}{l}
n \\
j
\end{array}\right)\left[F_{\Gamma}(\gamma)\right]^{j}
$$

\section{NUMERICAL RESULTS}

For correlated Nakagami- $m$ fading branches, the joint $\mathrm{CF}$ is given by [9, p. 359]

$$
\phi\left(t_{1}, \cdots, t_{n}\right)=|\mathbf{I}-j \mathbf{T S}|^{-m}
$$

where $\mathbf{I}$ is the identity matrix of size $n \times n,|\cdot|$ denotes the determinant, $\mathbf{T}=\operatorname{diag}\left\{t_{1}, t_{2}, \cdots, t_{n}\right\}, m \in[0.5, \infty)$, and $\mathbf{S}$ is a symmetric matrix with elements

$$
\mathbf{S}_{k, \ell}=\sqrt{\frac{\mathbf{R}_{k, \ell}}{m}} .
$$

In (11), $\mathbf{R}_{k, \ell} \triangleq \operatorname{Cov}\left(\Gamma_{k}, \Gamma_{\ell}\right)$ is the element in row $k$ and column $\ell$ of the correlation matrix $\mathbf{R}$.

\section{A. Constant Correlation Model}

The constant correlation model [2], [10] may be used either to approximate closely spaced antennas or to perform a firstorder analysis using the average value of the correlation coefficients for all the off-diagonal entries of the correlation matrix. Suppose we have a diversity receiver with an array of $n=3$ antennas placed at the vertices of an equilateral triangle. Then the branch gains are exchangeable Nakagami- $m$ rv's and the cdf can be obtained using (6). As an example, we consider the correlation matrix from [10]

$$
\mathbf{R}=\bar{\Gamma}\left[\begin{array}{lll}
1.0 & 0.6 & 0.6 \\
0.6 & 1.0 & 0.6 \\
0.6 & 0.6 & 1.0
\end{array}\right]
$$

where $\bar{\Gamma}$ denotes the average SNR.

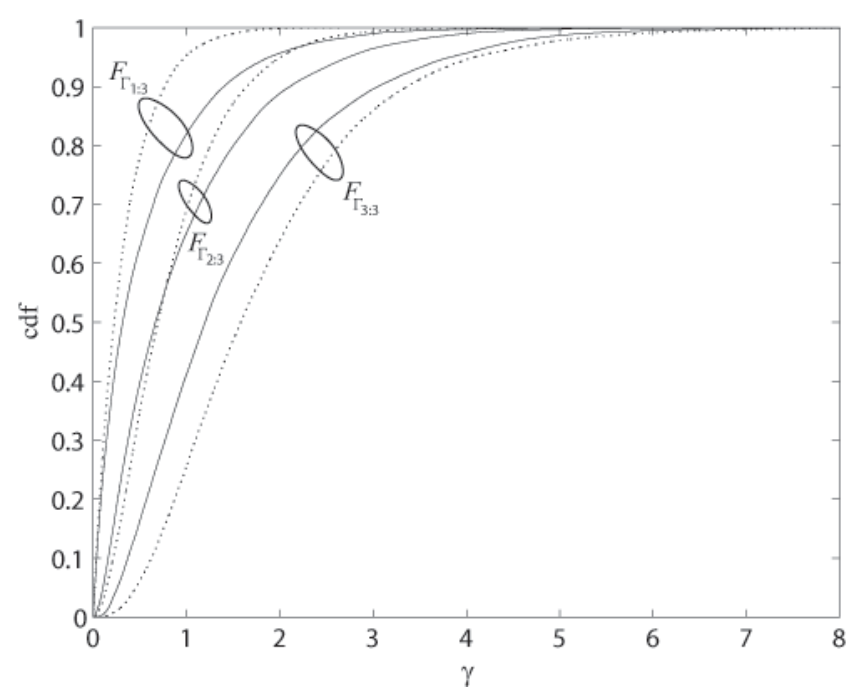

Fig. 1. Plot of cdf of $\Gamma_{1: 3}, \Gamma_{2: 3}$, and $\Gamma_{3: 3}$ with $m=1$ and $\bar{\Gamma}=1$ : independent (dotted) and correlated (solid) fading channels.

Fig. 1 shows the cdf of the $r$-th order statistic in a correlated Nakagami- $m$ fading environment (solid curves) with the correlation matrix in (12), $m=1$ and $\bar{\Gamma}=1$. The cdf curves for independent fading (dotted curves), obtained using (9), are also shown for comparison. When the highest SNR branch is selected (i.e. $\Gamma_{3: 3}$ ), the performance is better when the branches are independently fading; when the lowest SNR branch is selected (i.e. $\Gamma_{1: 3}$ ), correlated fading yields better performance. These observations are to be expected since correlation reduces the variability among the SNR values on the three branches.

The cdf of $\Gamma_{1: 3}$ is plotted in Fig. 2 for different values of $m$ with the correlation matrix in (12). As expected, it can be seen that the performance degrades as fading severity increases (i.e. $m$ decreases).

\section{B. Linear Model}

This type of correlation model applies to linear antenna arrays. We consider the correlation matrix example for a fiveelement array in [11]

$$
\mathbf{R}=\bar{\Gamma}\left[\begin{array}{lllll}
1.000 & 0.795 & 0.605 & 0.375 & 0.283 \\
0.795 & 1.000 & 0.795 & 0.605 & 0.375 \\
0.605 & 0.795 & 1.000 & 0.795 & 0.605 \\
0.375 & 0.605 & 0.795 & 1.000 & 0.795 \\
0.283 & 0.375 & 0.605 & 0.795 & 1.000
\end{array}\right]
$$




$$
F_{\Gamma_{r: n}}(\gamma)=\sum_{j=r}^{n}\left[(-1)^{j-r}\left(\begin{array}{l}
j-1 \\
r-1
\end{array}\right) \sum_{1 \leq i_{1}<\cdots<i_{j} \leq n} \prod_{k=1}^{j} F_{\Gamma_{i_{k}}}(\gamma)\right]
$$

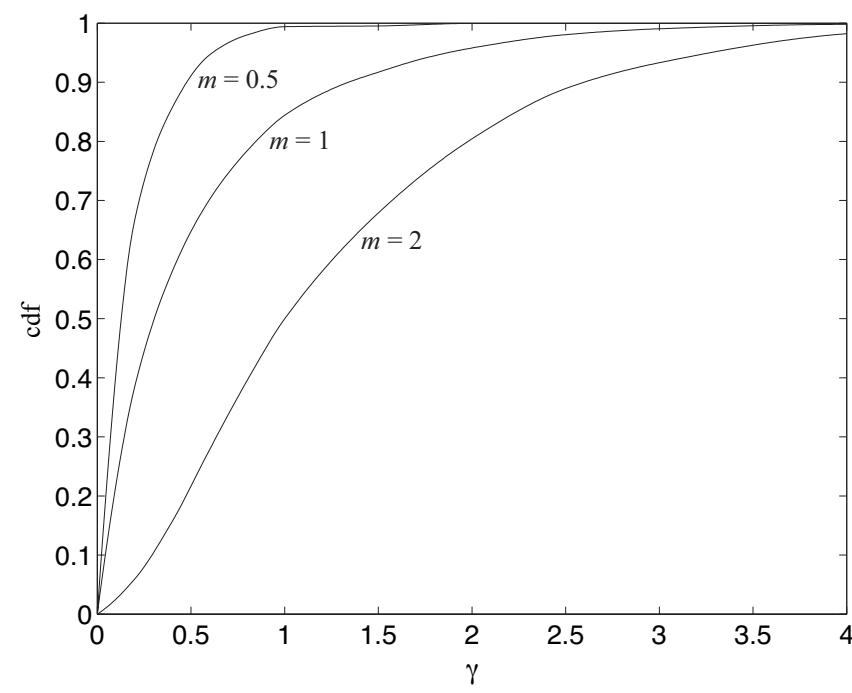

Fig. 2. Plot of cdf of $\Gamma_{1: 3}$ with $\bar{\Gamma}=1$ for different values of $m$.

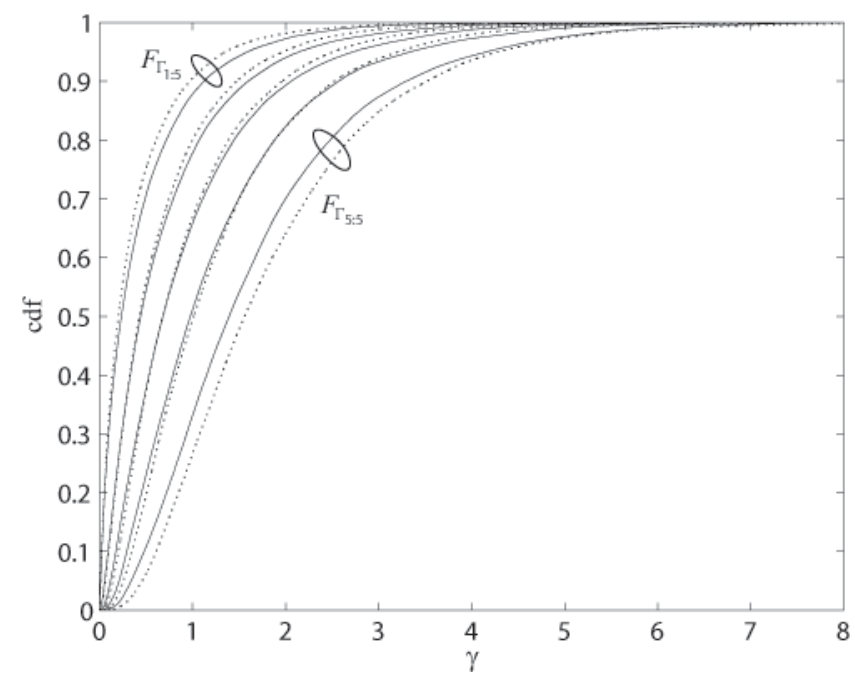

Fig. 3. Plot of cdf of $\Gamma_{r: 5}$ with $m=1$ and $\bar{\Gamma}=1$ for a linear model (solid) and its exchangeable approximation (dotted).

The correlation coefficient value decreases as the distance between array elements increases. In this case, the branch SNR rv's are not exchangeable and the cdf has to be obtained using (5). Fig. 3 shows the cdf for $\Gamma_{1: 5}, \Gamma_{2: 5}, \cdots, \Gamma_{5: 5}$ with the correlation matrix in (13), $m=1$ and $\bar{\Gamma}=1$ (solid curves). Approximate cdf curves (dotted curves), obtained using (6) and a $5 \times 5$ exchangeable matrix in which an average value of 0.514 is used for all off-diagonal entries, are also plotted. It can be seen that there is a significant difference between the exact and exchangeable approximation cdf curves for $\Gamma_{1: 5}$ and $\Gamma_{5: 5}$. On the other hand, the exchangeable approximation yields fairly accurate results for $\Gamma_{2: 5}, \Gamma_{3: 5}$ and $\Gamma_{4: 5}$.

\section{CONCLUSION}

In this letter, the cdf (and hence outage probability) of the $r$-th order branch SNR in correlated Nakagami- $-m$ fading was studied. The accuracy of a simple exchangeable approximation to reduce the computational load was examined. It would be interesting to extend the results to the performance analysis of generalized selection combining.

\section{ACKNOWLEDGMENT}

This work was supported in part by the Natural Sciences and Engineering Research Council (NSERC) of Canada under Grant OGP0001731 and by the UBC PMC-Sierra Professorship in Networking and Communications.

\section{REFERENCES}

[1] N. Kong and L. B. Milstein, "Average SNR of generalized diversity selection combining scheme," IEEE Commun. Lett., vol. 3, no. 3, pp. 57-59, Mar. 1999.

[2] M. K. Simon and M. S. Alouini, Digital Communication over Fading Channels, 2nd ed. New York: Wiley, 2005.

[3] Q. T. Zhang and H. G. Lu, "A general analytical approach to multibranch selection combining over various spatially correlated fading channels," IEEE Trans. Commun., vol. 50, no. 7, pp. 1066-1073, July 2002.

[4] R. K. Mallik and M. Z. Win, "Analysis of hybrid selection/maximal ratio combining in correlated Nakagami fading," IEEE Trans. Commun., vol. 50, no. 8, pp. 1372-1383, Aug. 2002.

[5] M. Z. Win et al., "On the SNR penalty of MPSK with hybrid selection/maximal ratio combining over i.i.d. Rayleigh fading channels," IEEE Trans. Commun., vol. 51, no. 6, pp. 1012-1023, June 2003.

[6] G. K. Karagiannidis, D. K. Zogas, and S. A. Kotsopoulos, "Performance analysis of triple selection diversity over exponentially correlated Nakagami-m fading channels," IEEE Trans. Commun., vol. 51, no. 8, pp. 1245-1248, Aug. 2003.

[7] H. A. David and H. N. Nagaraja, Order Statistics, 3rd ed. Hoboken, NJ: J. Wiley, 2003.

[8] A. Stuart and J. K. Ord, Kendall's Advanced Theory of Statistics, 6th ed. London, UK: Eward Arnold, 1994.

[9] M. D. Springer, The Algebra of Random Variables. New York: Wiley, 1979.

[10] G. K. Karagiannidis, D. K. Zogas, and S. A. Kotsopoulos, "An efficient approach to multivariate Nakagami- $m$ distribution using Green's matrix approximation," IEEE Trans. Wireless Commun., vol. 2, no. 5, pp. 883889, Sept. 2003.

[11] Q. T. Zhang, "Maximal-ratio combining over Nakagami fading channels with arbitrary branch covariance matrix," IEEE Trans. Veh. Technol., vol. 48, no. 4, pp. 1141-1150, Sept. 1999. 